\title{
Weak $\varepsilon$-Nets for Points on a Hypersphere
}

\author{
P. G. Bradford* and V. Capoyleas ${ }^{\dagger}$ \\ Max-Planck-Institut für Informatik, Im Stadtwald, \\ 66123 Saarbrücken, Germany \\ \{bradford, capoylea\}@mpi-sb.mpg.de
}

\begin{abstract}
A weak $\varepsilon$-net for a set of points $M$, is a set of points $W$ (not necessarily in $M$ ) where every convex set containing $\varepsilon|M|$ points in $M$ must contain at least one point in $W$. Weak $\varepsilon$-nets have applications in diverse areas such as computational geometry, learning theory, optimization, and statistics. Here we show that if $M$ is a set of points quasi-uniformly distributed on a unit sphere $S^{d-1}$, then there is a weak $\varepsilon$-net $W \subseteq \mathbb{R}^{d}$ of size $O(\log (1 / \varepsilon) \log (1 / \varepsilon))$ for $M$, where $k_{d}$ is exponential in $d$. A set of points $M$ is quasiuniformly distributed on $S^{d-1}$ if, for any spherical cap $\mathcal{C} \subseteq S^{d-1}$ with $\operatorname{Vol}(\mathcal{C}) \geq c_{1} /|M|$, we have
\end{abstract}

$$
c_{2} \operatorname{Vol}(\mathcal{C}) \leq|\mathcal{C} \cap M| \leq c_{3} \operatorname{Vol}(\mathcal{C})
$$

for three positive constants $c_{1}, c_{2}$, and $c_{3}$.

Further, we show that reducing our upper bound by asymptotically more than a $\log (1 / \varepsilon)$ factor directly implies the solution of a long unsolved problem of Danzer and Rogers.

\section{Introduction}

A weak $\varepsilon$-net for a set of points $M$ is a set of points $W$ (not necessarily in $M$ ) such that every convex set containing $\varepsilon|M|$ points in $M$ must contain at least one point in $W$.

Estimating the smallest possible size of weak $\varepsilon$-nets has attracted considerable attention recently. See, for example, [1], [6], [5], and [15]. Furthermore, many researchers have found applications of $\varepsilon$-nets in computational geometry, learning theory, optimization, and statistics, see Matoušek's chapter [14], for example.

\footnotetext{
* This author was supported by the ESPRIT Basic Research Actions Program, under Contract No. 7141 (project ALCOM II).

$\dagger$ This author's research was primarily done while at the Courant Institute, New York University. Partially supported by an IBM graduate fellowship, by NSF Grant CCR-9122103, and generously funded by the European Commission with a research fellowship of the Human Capital and Mobility (HCM) programme.
} 
In general, when $M$ can be an arbitrary point set in $\mathbb{R}^{d}$, the known bounds for the minimum size of $W$ are $\Omega(1 / \varepsilon)$ lower and $O\left(\left(1 / \varepsilon^{d}\right) \log ^{c_{d}}(1 / \varepsilon)\right)$ upper, where $c_{d}$ is an exponential function of $d$, see [6]. If $M$ is a planar set, there is a $O\left(1 / \varepsilon^{2}\right)$ upper bound, see [1].

There are better upper bounds for special cases. When $M$ is in convex position on the plane Chazelle et al. [6] give an upper bound of $O\left((1 / \varepsilon) \log ^{\log _{2} 3}(1 / \varepsilon)\right)$. When $M$ is a set of points uniformly (or quasi-uniformly) distributed on a circle, a bound of $O\left((1 / \varepsilon) 2^{\log ^{*}(1 / \varepsilon)}\right)$ obtained by Capoyleas [5], was later improved to the optimal $O(1 / \varepsilon)$ by Chazelle et al. [6]. As stated in the abstract, a set of points $M$ is quasiuniformly distributed on the unit sphere $S^{d-1}$ in $\mathbb{R}^{d}$, if for any spherical cap $\mathcal{C} \subseteq S^{d-1}$ with $\operatorname{Vol}(\mathcal{C}) \geq c_{1} /|M|$, we have $c_{2} \operatorname{Vol}(\mathcal{C}) \leq|\mathcal{C} \cap M| \leq c_{3} \operatorname{Vol}(\mathcal{C})$ for three constants $c_{1}, c_{2}, c_{3}>0$.

In this paper, we consider the special case when $M$ is a set of points quasi-uniformly distributed on the sphere $S^{d-1}$ for $d \geq 3$.

A famous problem, of Danzer and Rogers, asks how many points can stab all convex subsets of the unit square that have area $\varepsilon$. That is, how many points do we need in a set $W^{\prime}$ to be sure that all convex subsets of the unit sphere of area $\varepsilon$ contain a point of $W^{\prime}$. See Unsolved-Problem E14 in [8] and it is in the discussions in [1] and [3]. The presently known bounds for the size of the smallest possible stabbing set are $\Omega(1 / \varepsilon)$ lower and $O((1 / \varepsilon) \log (1 / \varepsilon))$ upper, but it is generally believed that the answer is likely to be nonlinear in $1 / \varepsilon$.

In Section 2 we review the necessary background for the rest of the paper. In Section 3 we show that $n$ points quasi-uniformly distributed on a $S^{d-1}$ sphere have weak $\varepsilon$-nets of size $O\left(\left(d^{2} \log (d) k_{d} / \varepsilon\right) \log \left(k_{d} / \varepsilon\right) \log (1 / \varepsilon)\right)$ and we give bounds for $k_{d}$. Finally, in Section 4 , we prove that any lower bound for Danzer and Roger's problem, is also a lower bound for the size of the weak $\varepsilon$-nets we are considering.

Special dependencies of the constants on $\varepsilon$ and $d$ will be noted as necessary.

\section{Foundations}

Let us recall some basic facts about $\varepsilon$-nets, for example, see the papers [12], [4], and [7], or the books [2], [16], and [18]. A range space $S$ is a pair $(X, R)$, where $X$ is a set and $R$ is a set of subsets of $X$ (that is, $R$ is a subset of the power set of $X$ ). The members of $X$ are elements. Members of $R$ are ranges. For some constant $\varepsilon, 1>\varepsilon>0$, a subset $N \subseteq X$ is called an $\varepsilon$-net of $S$ iff $N$ contains one point in each range $r \in R$ with $|r|>\varepsilon|X|$. It is easy to see that if $R$ is the power set of $X$, then an $\varepsilon$-net must have at least $(1-\varepsilon)|X|$ points.

A subset $A \subseteq X$ is said to be shattered by $R$, if every subset of $A$ can be obtained by intersecting $A$ with some range in $R$. That is, $A \subseteq X$ is said to be shattered by $R$ if $\{A \cap r: r \in R\}$ is the power set of $A$.

The Vapnik-Chervonenkis dimension of the range space (or $V C$-dimension, for short) is the cardinality of the largest shattered subset of $X$. If no such largest subset exists, the VC-dimension is infinite.

Proposition 1 gives a bound on the size of an $\varepsilon$-net, in terms of the VC-dimension of the underlining range space. For weak $\varepsilon$-nets however, no such proposition is proved. There is no obvious way to use the theory of range spaces and $\varepsilon$-nets, to bound the size of weak $\varepsilon$-nets, as we will do in this paper.

Vapnik and Chervonenkis first proved the following result in a more general context. 
Later using probabilistic methods, Haussler and Welzl [12] proved the next proposition in terms of $\varepsilon$-nets.

Proposition 1 [12]. Let $S=(X, R)$ be a range space of finite $V C$-dimension and let $1>\varepsilon>0$ and $|X| \gg 1 / \varepsilon{ }^{1}$ Then there exists an $\varepsilon$-net for $S$ of size $O((1 / \varepsilon) \log (1 / \varepsilon))$.

It is known that $O((1 / \varepsilon) \log (1 / \varepsilon))$ is independent of the size of $X$. Taking the dimension into account, Haussler and Welzl actually showed that for a range space $S$ of VC-dimension $d$, the $\varepsilon$-net for $S$ is of size at most

$$
\left\lceil\frac{8 d}{\varepsilon} \log \frac{8 d}{\varepsilon}\right\rceil
$$

and they gave a lower bound of $\Omega(d / \varepsilon)$. Later, Blummer et al. [4] showed that Haussler and Welzl's upper bound could be lowered. Further, Pach and Woeginger improved these results and so did Komlós (see [13], [17], and [14]). Together in [13] they gave the next upper bound and showed that it is tight

$$
\left\lceil(1+o(1)) \frac{8 d}{\varepsilon} \log \frac{1}{\varepsilon}\right\rceil
$$

for $d \geq 2$.

Given a range space $S=(X, R)$, we write $R^{*}$ to be the closure of the range $R$ under standard boolean (set-theoretic) formulas.

Proposition 2 [12], [10], [19]. Let $S=(X, R)$ be a range space offinite $V C$-dimension and let $k$ be a natural number. Suppose every range in $R^{*}$ can be expressed by a Boolean formula, involving $k$ elements of $R$. Then $S^{*}=\left(X, R^{*}\right)$ also has finite VC-dimension.

Furthermore, Haussler and Welzl actually showed that if the VC-dimension of $S$ is $d$, then the VC-dimension of the new range space $S^{*}$ is $O(d k \log (d k))$, see also [2], [18], and [14].

\section{The Construction}

In this section we prove the following theorem:

Theorem 3. Given a set $M$ of $n$ quasi-uniformly distributed points on $S^{d-1}$, then there exists a weak $\varepsilon$-net $W$ for $M$ such that

$$
|W|=O\left(\left(\frac{d^{2} \log (d) k_{d}}{\varepsilon}\right) \log \left(\frac{k_{d}}{\varepsilon}\right) \log \left(\frac{1}{\varepsilon}\right)\right),
$$

where $k_{d}$ is exponential in $d$.

\footnotetext{
${ }^{1}$ The relation $x \gg y$ means $x$ is larger than $y$ by at least a sufficiently large constant.
} 


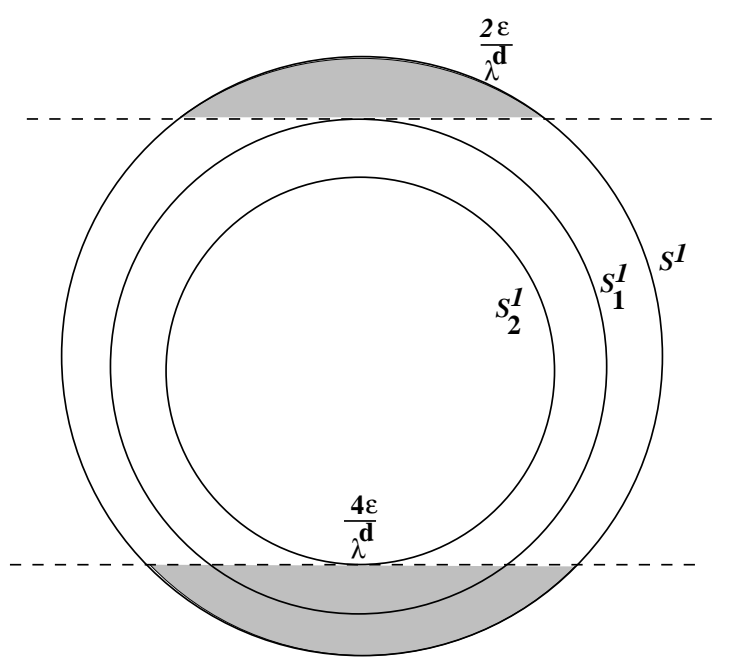

Fig. 1. Spheres $S^{1}, S_{1}^{1}$, and $S_{2}^{1}$ with their spherical caps shaded.

Let the sphere $S^{d-1}$ have unit surface volume and center $\mathcal{O}$. Take the sequence of concentric spheres with different radii,

$$
S_{1}^{d-1}, S_{2}^{d-1}, \ldots, S_{\left\lfloor\log \left(\lambda^{d} / \varepsilon\right)\right\rfloor-1}^{d-1}
$$

all centered at $\mathcal{O}$; and sphere $S_{i+1}^{d-1}$ is properly contained inside of the sphere $S_{i}^{d-1}$ for $i:\left\lfloor\log \left(\lambda^{d} / \varepsilon\right)\right\rfloor>i \geq 1$. And $S_{1}^{d-1}$ is inside $S^{d-1}$. (We will discuss the purpose of $\lambda^{d}$ shortly.)

Consider a hyperplane $\mathcal{H}_{i}$ tangent to $S_{i}^{d-1}$ that cuts off a spherical cap $\mathcal{C}_{i}$ from $S^{d-1}$. We choose the sizes of the spheres $S_{i}^{d-1}$ so that the volume of the spherical cap $\mathcal{C}_{i}$ is $2^{i} \varepsilon / \lambda^{d}$. See Fig. 1 .

In the rest of this section, we will prove that it is possible to put

$$
O\left(\left(d^{2} \log (d) k_{d} / \varepsilon\right) \log \left(k_{d} / \varepsilon\right)\right)
$$

points on each sphere $S_{i}^{d-1},\left\lfloor\log \left(\lambda^{d} / \varepsilon\right)\right\rfloor>i \geq 1$, so that the points on all the spheres together, form a weak $\varepsilon$-net for $M$, as stated in Theorem 3 .

The proof of Theorem 3 rests on the next two lemmas.

The intuition of the next lemma is that given a set of points whose maximum distance is large, then we can find a big subset contained in a ball that has a sufficiently large distance from at least one point.

Lemma 1. Let $M$ be a set of $n$ points with diameter $\delta$ in $\mathbb{R}^{d}$. For any real number $\lambda \geq 1$, there is a ball $B^{d}$ of radius $r=\delta \sqrt{d} / \lambda$ and center $p$ that contains at least $n / \lambda^{d}$ of the points in $M$ and there is a point $q \in M$ for which $\operatorname{dist}(p, q) \geq \delta / 2$.

Proof. Let $\delta$ be the diameter of $M$. Then we can show by induction on $d$ that $M$ is contained in a $d$-dimensional cube with sides of size $\delta$. Therefore it can be covered by 
$\lambda^{d}$ cubes with sides of size $\delta / \lambda$. By the pigeon-hole principle at least one of these cubes must contain at least $n / \lambda^{d}$ points of $M$.

Let $B^{d}$ be the smallest ball containing this cube and let $B^{d}$ have center $p$. Consider two points in $M$ which are at a distance of $\delta$ from each other. Of these two points, let $q$ be the one whose distance from $p$ is greater. Clearly, $\operatorname{dist}(p, q) \geq \delta / 2$ and in the same way we can compute the bound on $r$ using the Pythagorean theorem.

The next lemma is used for showing that we will always have a sufficiently large subset of a convex set that intersects some inner sphere $S_{i}^{d-1}$. More precisely, the central projections we are using are roughly volume and area preserving.

Lemma 2. Let $S^{d-1}$ and $T^{d-1}$ be two concentric spheres centered at $\mathcal{O}$ and $T^{d-1}$ is inside $S^{d-1}$. Let $\mathcal{H}$ be a hyperplane tangent to $T^{d-1}$ cutting off a spherical cap $\mathcal{C}$ from $S^{d-1}$ with center $p$ and diameter $\delta$. Let $q \in S^{d-1}$ be so that $\operatorname{dist}(p, q) \geq k \delta$, where $k>1$, and it is sufficiently large. Choose any two points $a, b \in \mathcal{C}$, where the lines $a-q$ and $b-q$ intersect $\mathcal{H}$ and $T^{d-1}$, at the pairs of points $\left(a^{\prime}, b^{\prime}\right)$ and $\left(a^{\prime \prime}, b^{\prime \prime}\right)$, respectively. See Fig. 2. Then the distances $\operatorname{dist}(a, b), \operatorname{dist}\left(a^{\prime}, b^{\prime}\right)$, and $\operatorname{dist}\left(a^{\prime \prime}, b^{\prime \prime}\right)$ are roughly equal; in particular,

$$
1-\frac{1}{k}<\frac{\operatorname{dist}(a, b)}{\operatorname{dist}\left(a^{\prime}, b^{\prime}\right)} \quad \text { and } \quad \frac{\operatorname{dist}(a, b)}{\operatorname{dist}\left(a^{\prime \prime}, b^{\prime \prime}\right)}<1+\frac{1}{k}
$$

Proof. Intersect $S^{d-1}, T^{d-1}$, and $\mathcal{H}$ by the two-dimensional plane determined by the points $a, b$, and $q$. Now obtain an equivalent plane geometry problem. The required conditions follow from the law of similar triangles from elementary geometry. That is, two similar triangles have their corresponding angles equal. Further, edge lengths of two similar triangles are different by the same multiplicative constant.

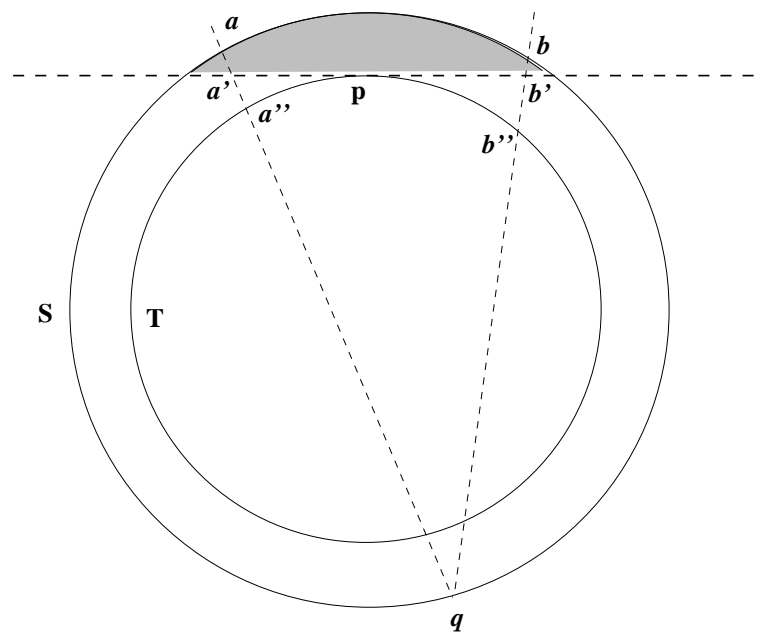

Fig. 2. Spheres $S^{d-1}$ and $T^{d-1}$ with $\mathcal{C}$ at the top and the points $a, b, a^{\prime}, b^{\prime}, a^{\prime \prime}$, and $b^{\prime \prime}$ shown. 
These similar triangles bound the distance along the arcs of the spheres. This completes the proof.

Suppose we have a set $M$ of points that are quasi-uniformly distributed on the sphere $S^{d-1}$. Our goal is to place the points of $W$ on all of the spheres $S_{i}^{d-1}$ for $i:\left\lfloor\log \left(\lambda^{d} / \varepsilon\right)\right\rfloor>$ $i \geq 1$, so as to stab the convex hull of every $K \subseteq M$ with $|K| \geq \varepsilon|M|$.

Consider such a set of points $K$ and choose a large constant $\lambda^{d}$ for some $\lambda>1$. By Lemma 1 , and for sufficiently large $\lambda$, there is some $K^{\prime} \subseteq K$, where $\left|K^{\prime}\right| \geq \varepsilon|K| / \lambda^{d}$ and $q \in K$, such that $K^{\prime}$ is in some spherical cap $\mathcal{C}$ of $S^{d-1}$ and also in a ball $B$ of radius $r=\delta \sqrt{d} / \lambda$ where $\delta$ is the diameter of the set $K$. The spherical cap $\mathcal{C}$ has diameter $\delta_{1}$, base hyperplane $\mathcal{H}$, center $p$, and the value of $\lambda$ can be chosen so that $\operatorname{dist}(p, q)>k_{1} \delta_{1}$, where $k_{1}$ is large. (That is, $k_{1} \delta_{1} \gg \delta / 2$.)

We can increase $\delta_{1}$ by a factor of less than 2 and have $\mathcal{H}$ touch one of the spheres in our sequence, call it $T^{d-1}$, without violating any of the other conditions already established. This is because sphere $S_{i}^{d-1}$ and hyperplane $\mathcal{H}_{i}$ cut off a spherical cap $\mathcal{C}_{i}$ with $2^{i} \varepsilon / \lambda^{d}$ surface volume, where we can adjust the value of $\lambda$ to make it so that $\delta_{1}$ is changed appropriately. Since each successive hyperplane cuts off double the volume of the previous one, then the diameter of each successive spherical cap must be less than double. (Note that $k_{1}$ will still be large.)

We arrange the points in $W$ so that $\operatorname{conv}\left(K^{\prime} \cup\{q\}\right)$ is stabbed by (that is, it contains) at least one of the points of $W$ that we will distribute on $T^{d-1}$. To this end, consider the set,

$$
\mathcal{I}=\operatorname{conv}\left(K^{\prime} \cup\{q\}\right) \cap T^{d-1} .
$$

A spherical simplex on a sphere $S^{d-1}$ is the intersection of $S^{d-1}$ and $d$ half-spaces.

Claim 1. Let $c$ be a large constant independent of $d$. The set $\mathcal{I}$ contains a spherical simplex $\mathcal{A}$ with $\operatorname{Vol}(\mathcal{A})>\varepsilon /\left(2^{d} d ! \lambda^{d} c\right)$.

Proof. Let $K_{\mathcal{H}}^{\prime}$ denote the image of $K^{\prime}$ on $\mathcal{H}$, under a central projection through the point $q$. By Lemma 2, this projection does not affect distances by more than a small constant factor. Clearly, $\operatorname{conv}\left(K_{\mathcal{H}}^{\prime}\right) \subseteq \operatorname{conv}\left(K^{\prime} \cup\{q\}\right)$ and $\operatorname{Vol}\left(\operatorname{conv}\left(K_{\mathcal{H}}^{\prime}\right)\right) \geq$ $\varepsilon /\left(\lambda^{d} c^{\prime}\right)$ for some constant $c^{\prime}$. But then, $\operatorname{conv}\left(K_{\mathcal{H}}^{\prime}\right)$ contains a simplex $\mathcal{A}$, with $\operatorname{Vol}(\mathcal{A})>$ $\varepsilon /\left(c^{\prime \prime} 2^{d} d ! \lambda^{d}\right)$ for some constant $c^{\prime \prime}$.

A convex body in $d$ dimensions of volume $V=\varepsilon /\left(\lambda^{d} c^{\prime}\right)$ must contain an elipsoid of volume at least $V / 2^{d}$. This follows from the existence of the Löwner-John elipsoid, see [11], for example. Such an elipsoid, and therefore our convex body itself, contains a simplex of volume $V /\left(2^{d} d !\right)$.

Centrally project the mapping from $A$ through $q$, onto $T^{d-1}$, to obtain $\mathcal{A} \subseteq \mathcal{I}$. $\mathcal{A}$ is a spherical simplex, and by Lemma 2, we know that $\operatorname{Vol}(\mathcal{A})>\varepsilon /\left(c 2^{d} d ! \lambda^{d}\right)$.

Next, we show how to place

$$
O\left(\left(\frac{d^{2} \log (d) 2^{d} d ! \lambda^{d}}{\varepsilon}\right) \log \left(\frac{2^{d} d ! \lambda^{d}}{\varepsilon}\right)\right)
$$

points of $W$ on $T^{d-1}$, so that they stab every spherical simplex on $T^{d-1}$ of volume $\varepsilon / c$. 
Let us construct range spaces $S=(X, R)$ and $S^{*}=\left(X, R^{*}\right)$ as follows. Let $X$ be a set of points quasi-uniformly distributed on $T^{d-1},|X| \gg 1 / \varepsilon$. Let $R$ be all the sets that are intersections of $X$ with half-spaces. Let $R^{*}$ be the sets that are intersections of $X$ with spherical simplices. The range space $S$ has VC-dimension roughly $d$; it can be easily seen that a set of at most $d+1$ points in $\mathbb{R}^{d}$ can be shattered with half-spaces. By Proposition 2 the VC-dimension of $S^{*}$ is

$$
O(d k \log (d k))=O\left(d^{2} \log d\right)
$$

since $k=d$.

Clearly, every spherical simplex on $T^{d-1}$ of volume $\varepsilon /\left(2^{d} d ! \lambda^{d} c\right)$ contains at least $\varepsilon|X| /\left(2^{d} d ! \lambda^{d} c^{\prime}\right)$ points of $X$ for some constant $c^{\prime}$, by the quasi-uniform distribution of the points of $X$. By Proposition $1, S^{*}$ has an $\left(\varepsilon / 2^{d} d ! \lambda^{d} c^{\prime \prime}\right)$-net $N$, for some constant $c^{\prime \prime}$ and where

$$
|N|=O\left(\left(\frac{d^{2} \log (d) 2^{d} d ! \lambda^{d}}{\varepsilon}\right) \log \left(\frac{2^{d} d ! \lambda^{d}}{\varepsilon}\right)\right)
$$

Hence $N$ stabs all spherical simplices $\mathcal{A}$ on $T^{d-1}$, with $\operatorname{Vol}(\mathcal{A}) \geq \varepsilon /\left(2^{d} d ! \lambda^{d} c\right)$.

We can finish the construction of $W$, simply by repeating this construction of $N$ for each $S_{i}^{d-1}$. We have to iterate this process at most $\log \left(\lambda^{d} / \varepsilon\right)$ times. Letting $k_{d}=2^{d} d ! \lambda^{d}$ for some constant $\lambda>1$, the bound in Theorem 3 can be expressed tightly as

$$
O\left(\left(\frac{d^{2} \log (d) k_{d}}{\varepsilon}\right) \log \left(\frac{k_{d}}{\varepsilon}\right) \log \left(\frac{1}{\varepsilon}\right)\right) .
$$

We can reduce the factor $2^{d} d$ ! in $k_{d}$ by a little, but $k_{d}$ will still be exponential.

\section{Relation to a Problem of Danzer and Rogers}

In this section we show that $|W|$, the size of the weak $\varepsilon$-net for points quasi-uniformly distributed on a sphere, is asymptotically at least as big as the size of the smallest stabbing set for Danzer and Rogers's problem.

Danzer and Rogers posed the following problem [9]:

Given a unit square how many points are necessary to hit every convex set of area $\varepsilon$ contained in the square?

See Unsolved-Problem E14 in [8].

Theorem 4. Let $M$ be a set of points quasi-uniformly distributed on a three-dimensional sphere $S^{2}$ and let $W$ be a weak $\varepsilon$-net for $M, 0<\varepsilon<1$. Let $W^{\prime}$ be a set of points in a unit square, such that every convex subset of the square with area $\varepsilon$ contains a point in $W^{\prime}$. Then $|W|(\varepsilon)=\Omega\left(\left|W^{\prime}\right|(\varepsilon)\right)$.

Proof. Consider a sphere $S^{2}$ with big constant radius $R$ and center $\mathcal{O}$, and a horizontally placed unit square touching the sphere at the point vertically above the center $\mathcal{O}$. Let $M$ 
be quasi-uniformly distributed on $S^{2}$ and let $W$ be a weak $\varepsilon$-net for it. Centrally project $W$ onto the square, to obtain $W^{\prime}, \mathcal{O}$ being the center of the projection.

Now consider any convex subset of the square, call it $\mathcal{C}$. Clearly, $\mathcal{I}=S^{2} \cap \operatorname{conv}(\mathcal{C} \cup \mathcal{O})$ contains roughly $\operatorname{area}(\mathcal{C})|M| / 4 \pi R^{2}$ points of $M$ (see Lemma 2 ). If $\operatorname{area}(\mathcal{C}) \geq \varepsilon c R, c$ being large, then $\operatorname{conv}(\mathcal{I})$ must contain a point of $W$ and $\operatorname{since} \operatorname{conv}(\mathcal{I}) \subseteq \operatorname{conv}(\mathcal{I} \cup \mathcal{O})$, $\mathcal{C}$ must contain a point of $W^{\prime}$. Therefore $W^{\prime}$ stabs all convex subsets of the square that have area $\varepsilon c R$.

This theorem points out that if we shave off more than an asymptotic $\log (1 / \varepsilon)$ factor from the weak $\varepsilon$-net for the sphere $S^{2}$, then we can shave off more than a constant from the solution of Danzer and Rogers's problem.

Let us briefly consider the case where we allow $M$ to be arbitrarily distributed on $S^{d-1}$. By a simple generalization of the above method, we can show that any upper bound on the size of the weak $\varepsilon$-net of $M$, is also an upper bound on the size of weak $\varepsilon$-nets of points arbitrarily distributed in $\mathbb{R}^{d-1}$.

The best-known bound for the size of weak $\varepsilon$-nets of points arbitrarily distributed in $\mathbb{R}^{d-1}$ is $O\left(\left(1 / \varepsilon^{d-1}\right) \log ^{c_{d-1}}(1 / \varepsilon)\right)$ by Chazelle et al. [6]. This bound is considered hard to improve.

\section{Conclusions}

As we noted in the Introduction, the best upper bound for a weak $\varepsilon$-net for any set $M$ in $\mathbb{R}^{d}$ is $O\left(\left(1 / \varepsilon^{d}\right) \log ^{c_{d}}(1 / \varepsilon)\right)$ where $c_{d}$ is exponential in $d$ and is due to Chazelle et al. [6]. Here we show how to construct a weak $\varepsilon$-net for points quasi-uniform distribution on a sphere $S^{d-1}$ with $O\left(\left(\left(d^{2} \log (d) k_{d}\right) / \varepsilon\right) \log \left(k_{d} / \varepsilon\right) \log (1 / \varepsilon)\right)$ points in our weak $\varepsilon$-net for $k_{d}$ exponential in $d$. Our discussion in Section 4 leaves little hope for improving our bound by more than a $\log (1 / \varepsilon)$ factor or for generalizing our bound for points arbitrarily distributed on the sphere.

\section{References}

1. N. Alon, I. Bárány, Z. Füredi, and D. Kleitman. Point selections and weak $\varepsilon$-nets for convex hulls. Combin. Probab. Comput. 1 (1992), 189-200.

2. N. Alon and J. Spencer. The Probabilistic Method. Wiley, New York, 1992.

3. J. Beck and W. Chen. Irregularities of Distributions. Cambridge Tracts in Mathematics, Vol. 89. Cambridge University Press, Cambridge, 1987.

4. A. Blumer, A. Ehrenfeucht, D. Haussler, and M. Warmuth. Learnability and the Vapnik-Chervonenkis dimension. J. Assoc. Comput. Mach. 36 (1989), 929-965.

5. V. Capoyleas. An upper bound for weak $\varepsilon$-nets of points on a circle. In: Intuitive Geometry (K. Böröczky and G. Fejes Tóth, eds.). Coll. Math. Soc. J. Bolyai, vol. 63. North-Holland, 1994, pp. 63-66.

6. B. Chazelle, H. Edelsbrunner, M. Grigni, L. Guibas, M. Sharir, and E. Welzl. Improved bounds on weak $\varepsilon$-nets for convex sets. In: Proceedings of the Symposium on the Theory of Computing (STOC), vol. 25. ACM Press, New York, 1993, pp. 495-504.

7. B. Chazelle and E. Welzl. Quasi-optimal range searching and VC-dimensions. Discrete Comput. Geom. 4 (1989), 467-490. 
8. H. T. Croft, K. J. Falconer, and R. K. Guy. Unsolved Problems in Geometry. Unsolved Problems in Intuitive Mathematics, vol. 2. Springer-Verlag, New York, 1991.

9. E. Danzer. Personal Communication.

10. R. Dudley. Central limit theorems for empirical measures. Ann. Probab. 6 (1978), 899-929.

11. M. Grötschel, L. Lovász, and A. Schrijver. Geometric Algorithms and Combinatorial Optimization. Algorithms and Combinatorics, vol. 2. Springer-Verlag, New York, 1988.

12. D. Haussler and E. Welzl. $\varepsilon$-Nets and simplex range queries. Discrete Comput. Geom. 2 (1987), 127-151.

13. J. Komlós, J. Pach, and G. Woeginger. Almost tight bounds for $\varepsilon$-nets. Discrete Comput. Geom. 7 (1992), $163-173$.

14. J. Matoušek. Epsilon-nets and computational geometry. In: New Trends in Discrete and Computational Geometry (J. Pach, ed.), Chapter 3. Algorithms and Combinatrics, vol. 10. Springer-Verlag, New York, 1993.

15. J. Matoušek, R. Seidel, and E. Welzl. How to net a lot with a little: Small $\varepsilon$-nets for disks and halfspaces. In: Proceedings of ACM Symp. Comput. Geom., vol. 6. ACM Press, New York, 1990, pp. 16-22.

16. K. Mulmuley. Computational Geometry-An Introduction Through Randomized Algorithms. Prentice Hall, Englewood Cliffs, NJ, 1994.

17. J. Pach and G. Woeginger. Some new bounds for epsilon-nets. In: Proceedings of the 6th Comput. Geom. Conf. ACM Press, New York, 1990, pp. 10-15.

18. M. Sharir and P. Agarwal. Davenport-Schinzel Sequences and Their Geometric Applications. Cambridge University Press, Cambridge, 1995.

19. R. Wenocur and R. Dudley. Some Vapnik-Chervonenkis classes. Discrete Math. 33 (1981), 313-318.

Received April 12, 1995, and in revised form May 8, 1995. 